Check for updates

Cite this: RSC Adv., 2019, 9, 20989

\title{
Nonlocal effects on the structural transition of gold clusters from planar to three-dimensional geometries $\dagger$
}

\begin{abstract}
Ping Wu, Qingxiu Liu and Gang Chen (iD *
Conventional density functional theory calculations heavily bias planar structures in gold clusters, failing to predict the structural transition from planar to three-dimensional geometries in experimentally detected gold species. Inspired by recent progress in calculating the defect energies of coinage metals with nonlocal effect-enhanced hybrid functionals, we have studied nonlocal effects in gold clusters. Although the hybrid functional was accurate for bulk gold, it heavily biased the planar structure for gold clusters. By including dispersive interactions into semilocal density functional calculations, we obtained an accurate vacancy formation energy of $0.72 \mathrm{eV}$ for bulk gold along with the correct structural transition for gold clusters. The transition was found to occur at $\mathrm{Au}_{12}{ }^{-}$for gold anions and $\mathrm{At} \mathrm{Au}_{8}{ }^{+}$for gold cations, agreeing very well with the experimental results. For neutral gold clusters, we found the transition to occur at $\mathrm{Au}_{10}$, indicating the need for experimental verification. The results show the importance of nonlocal effects in the study of gold clusters, calling for further comprehensive theoretical and experimental studies to evaluate nonlocal effects in $\mathrm{Au}$ and other precious metals.
\end{abstract}

Received 21st March 2019

Accepted 22nd June 2019

DOI: $10.1039 / c 9 r a 02202 c$

rsc.li/rsc-advances biases the 2D geometries of gold clusters, making the assignment of the 2D-3D crossover cluster difficult solely based on density functional theory (DFT) calculations. For example, for $\mathrm{Au}_{16}{ }^{-}, \mathrm{Au}_{12}{ }^{-}$, and $\mathrm{Au}_{8}{ }^{+}$, DFT calculations using the generalized gradient approximation with Perdew, Burke, and Ernzerhof (GGA-PBE) functional ${ }^{30}$ favor the $2 \mathrm{D}$ geometries, whereas experimental studies indicate that the 3D configurations are preferred. Currently, one usually calculates the properties of several low-lying isomers and compares them with experimental observations to assign the ground-state geometry. However, the standard semilocal functionals heavily bias the $2 \mathrm{D}$ configurations for gold clusters, a problem that still puzzles theoretical researchers. The PBEsol revised to enhance surface energy $^{31}$ along with the meta-GGA functional of the Tao-Perdew-Staroverov-Scuseria (TPSS) formalism ${ }^{32}$ and its revised functional revTPSS ${ }^{33}$ can improve the overall accuracy; however, the experimental results of gold clusters are still not well accounted for. As evidenced by the joint experiment/theory studies on clusters of gold anions conducted by Lechtken et al. ${ }^{34}$ and Johansson et al., ${ }^{35}$ the TPSS and PBEsol functionals still tend to overestimate the stabilities of the $2 \mathrm{D}$ geometries. In addition, PBEsol, TPSS, and revTPSS cannot consistently calculate the vacancy formation energies of coinage metals. Recently, accurate vacancy formation energies of 1.06, 0.94, and $0.72 \mathrm{eV}$ for $\mathrm{Cu}, \mathrm{Ag}$, and $\mathrm{Au}$ metals, respectively, were reported; $;^{36,37}$ however, the respective energies were calculated to be $1.27,1.01$, and $0.57 \mathrm{eV}$ with PBEsol $^{38}$ and 1.50, 1.25, and $0.92 \mathrm{eV}$ with revTPSS. ${ }^{39}$ As discussed later in this paper, we
Department of Physics, University of Jinan, Shandong 250022, China. E-mail: ss_cheng@ujn.edu.cn

$\dagger$ Electronic supplementary information (ESI) available. See DOI: $10.1039 / \mathrm{c} 9 \mathrm{ra02202c}$ 
calculated them to be $1.39,1.12$, and $0.76 \mathrm{eV}$, respectively, using the TPSS functional.

Recently, Chen and co-workers ${ }^{37}$ obtained accurate vacancy formation energies for bulk $\mathrm{Cu}, \mathrm{Ag}$, and $\mathrm{Au}$ by including nonlocal exchange interactions with the Heyd, Scuseria, and Ernzerhof (HSE) hybrid functional. ${ }^{40}$ The effects of the nonlocal exchange interaction were also found in Zhang et al. ${ }^{41}$ HSE calculations for $\mathrm{Cu}-\mathrm{Au}$ intermetallic alloys. By including the nonlocal correlation energies in DFT calculations, Aguado et al. ${ }^{42}$ and Fernández et al. ${ }^{43}$ obtained improved results for $\mathrm{Na}$, $\mathrm{Au}$, and $\mathrm{Hg}$ clusters. However, for the typical $\mathrm{Au}_{12}{ }^{-}$cluster, the calculations carried out by Aguado et al. still biased the 2D structure in comparison to the experimental conclusions. By using the HSE functional with $\alpha=0.4$, following Chen's choice for bulk gold, ${ }^{37}$ our calculations show that the method of enhancing nonlocal exchange effects cannot properly treat gold clusters. With the goal of identifying a computational method that is appropriate for both bulk gold and gold clusters, we carried out DFT calculations while compensating the nonlocal effects by considering the dispersive interactions through the Tkatchenko-Scheffler dispersive (TSD) approach ${ }^{44}$ which is based on the mean-field ground-state electronic density from DFT calculations and has only one empirical parameter for tuning the onset of the dispersive correction in the DFT energy. Using the vacancy formation energy of bulk gold as a reference, we first tuned this parameter and then studied the anionic, neutral, and cationic gold clusters. Interestingly, the obtained results agree very well with the experimental data, indicating the validity of the TSD-DFT method for studying both bulk and nanostructured gold materials.

\section{Computational details}

Our calculations were carried out using spin-polarized DFT implemented in the Vienna ab initio simulation package (VASP) ${ }^{45}$ The projector augmented-wave (PAW) method with the wavefunction expressed using a planewave basis set was employed. Relativistic effects were included using the scalar relativistic approach. A cutoff energy of $500 \mathrm{eV}$ was used to account for the properties of bulk gold and gold clusters. The convergence tolerance of the electronic properties and atomic relaxation were set to $0.001 \mathrm{meV}$ and $5 \mathrm{meV} \AA^{-1}$, respectively. To eliminate the interaction between neighboring vacancies in bulk gold, a fcc-type supercell containing 64 metal atoms was used. The lattices were first optimized using different exchange-correlation energy functionals with a $7 \times 7 \times 7$ Monkhorst-Pack $k$-point mesh. ${ }^{46}$ For comparison with Chen's HSE studies, ${ }^{37} \mathrm{Cu}, \mathrm{Ag}$, and Au metals were first calculated as reference to examine the validity of the TSD-PBE method. The calculation results obtained using PBE, PBEsol, TPSS, revTPSS, and TSD-PBE are provided in Table 1. For the optimized fcc-type supercell, we calculated the total energy $E_{64}$ using a $13 \times 13 \times$ 13 Monkhorst-Pack $k$-point mesh. Subsequently, one of the atoms was removed, and the other atoms were fully relaxed. The obtained total energy $E_{63}$ for the vacancy-defected bulk was used to calculate the vacancy formation energy $E_{\mathrm{v}}$ using the following formula:

$$
E_{\mathrm{v}}=E_{63}+\mu-E_{64},
$$

where $\mu$ is the chemical potential corresponding to the mean energy of a metal atom in the corresponding metal crystal. We also calculated the $\mathrm{Au}_{2}{ }^{-}, \mathrm{Au}_{2}$, and $\mathrm{Au}_{2}{ }^{+}$dimers as references to validate the TSD-PBE method. In our calculations, a cubic supercell with an edge length of $20 \AA$ was used to study the gold dimers and other gold clusters. This supercell is sufficiently large to ignore the interactions with their periodic images. The bond lengths and binding energies of the gold dimers are presented in Table 2. Based on the data shown in Tables 1 and 2, the TSD-PBE method can be used to treat both bulk gold and gold clusters.

In addition to considering the structures of gold clusters published in the literature, we searched the geometric structures and selected low-energy structural configurations via global minimum structural searching with an efficient evolutionary algorithm implemented in the Universal Structure Predictor: Evolutionary Xtallography (USPEX) ${ }^{49-52}$ interfaced with VASP code. This method can efficiently predict geometric structures for crystalline material, nanostructure, polymer, surface, and interface systems containing as many as $\sim 200$ atoms per cell. A certain number of structures were randomly produced to ensure the unbiased sampling of the energy landscape, and the generated structures were then subjected to firstprinciples structural optimization. The calculated total energies were used to select the low-lying isomers. In the evolutionary process of the structural search, the population of $30 \%$ of the candidate structures with low total energies were evolved over successive generations of random variation and selection.

\section{Results and discussion}

\subsection{Calculations of $\mathrm{Au}_{n}{ }^{-}$clusters with semilocal functionals}

We began our calculations using $\mathrm{Au}_{n}{ }^{-}(n \leq 16)$ clusters by combining the global minimum structural search with firstprinciples calculations using different semilocal functionals. The semilocal density functional PBE, ${ }^{30}$ the PBEsol functional revised for densely packed solids and their surfaces, ${ }^{31}$ the advanced semilocal functional meta-GGA TPSS, ${ }^{32}$ and the revTPSS functional constructed by restoring the second-order gradient expansion for exchange over a wide range of densities $^{33}$ were used. First, we studied the 2D and 3D structures for each cluster. Among the studied 2D geometries, the one with the lowest energy was selected, as schematically illustrated in the ESI. $\dagger$ Similarly, the corresponding lowest-energy 3D geometry among all the calculated non-planar structures was selected for each cluster and is shown in the ESI. $\dagger$ Fig. S1-S4 $\uparrow$ show the structures obtained using the PBE, PBEsol, TPSS, and revTPSS functionals, respectively. We then calculated their relative energies $\left(E_{\mathrm{r}}\right)$ as

$$
E_{\mathrm{r}}=E_{3 \mathrm{D}}-E_{2 \mathrm{D}}
$$

where $E_{2 \mathrm{D}}$ and $E_{3 \mathrm{D}}$ are the total energies of the corresponding 2D and 3D structures, respectively. The conventional PBE functional cannot accurately describe small gold clusters. As 
Table 1 Lattice constants a (Å) and vacancy formation energies $E_{v}(e V)$ of $C u, A g$, and Au fcc crystals calculated with the PBE, PBEsol, TPSS, revTPSS, and TSD-PBE methods. The experimental and HSE data are cited from ref. 36 and 37, respectively. EP stands for the empirical parameter $\alpha$ for the hybridization ratio in HSE or $S_{R}$ as the scaling factor in the Fermi-type damping function of the TSD-vdW method

\begin{tabular}{|c|c|c|c|c|c|c|c|c|c|}
\hline \multirow[b]{2}{*}{ Method } & \multicolumn{3}{|l|}{$\mathrm{Cu}$} & \multicolumn{3}{|l|}{$\mathrm{Ag}$} & \multicolumn{3}{|l|}{$\mathrm{Au}$} \\
\hline & $a$ & $E_{\mathrm{v}}$ & $\mathrm{EP}$ & $a$ & $E_{\mathrm{v}}$ & EP & $a$ & $E_{\mathrm{v}}$ & $\mathrm{EP}$ \\
\hline Exp. & 3.615 & 1.06 & - & 4.069 & 0.94 & & 4.077 & - & \\
\hline PBE & 3.635 & 1.059 & - & 4.148 & 0.790 & & 4.157 & 0.390 & \\
\hline PBEsol & 3.569 & 1.230 & - & 4.052 & 0.999 & & 4.082 & 0.568 & \\
\hline TPSS & 3.587 & 1.391 & - & 4.089 & 1.124 & & 4.113 & 0.756 & \\
\hline revTPSS & 3.561 & 1.487 & - & 4.052 & 1.228 & & 4.077 & 0.870 & \\
\hline HSE & 3.623 & 1.06 & 0.10 & 4.142 & 0.94 & 0.25 & 4.124 & 0.72 & 0.40 \\
\hline TSD-PBE & 3.634 & 1.064 & 3.989 & 4.129 & 0.944 & 1.287 & 4.143 & 0.724 & 0.796 \\
\hline
\end{tabular}

Table 2 Bond length $(\AA)$ and binding energy (eV) data from the studied gold dimer. The empirical parameter in HSE was chosen as $\alpha=0.4$ following Chen. ${ }^{37}$ For the TSD-PBE calculation, the Fermi-type damping parameter $s_{R}=0.796$ obtained in the calculation of the vacancy formation energy of bulk gold was adopted. The experimental data are taken from ref. 47 and 48

\begin{tabular}{|c|c|c|c|c|c|c|}
\hline \multirow[b]{2}{*}{ Method } & \multicolumn{2}{|l|}{$\mathrm{Au}_{2}{ }^{-}$} & \multicolumn{2}{|l|}{$\mathrm{Au}_{2}$} & \multicolumn{2}{|l|}{$\mathrm{Au}_{2}^{+}$} \\
\hline & $R$ & $E$ & $R$ & $E$ & $R$ & $E$ \\
\hline PBE & 2.624 & 1.903 & 2.509 & 2.337 & 2.601 & 2.544 \\
\hline PBEsol & 2.574 & 2.140 & 2.473 & 2.596 & 2.560 & 2.758 \\
\hline HSE & 2.624 & 1.682 & 2.507 & 1.935 & 2.623 & 2.093 \\
\hline TPSS & 2.609 & 1.896 & 2.498 & 2.315 & 2.594 & 2.479 \\
\hline revTPSS & 2.591 & 1.994 & 2.488 & 2.429 & 2.579 & 2.562 \\
\hline TSD-PBE & 2.626 & 1.907 & 2.512 & 2.345 & 2.612 & 2.566 \\
\hline Expt. & 2.582 & 1.92 & 2.472 & 2.29 & & \\
\hline
\end{tabular}

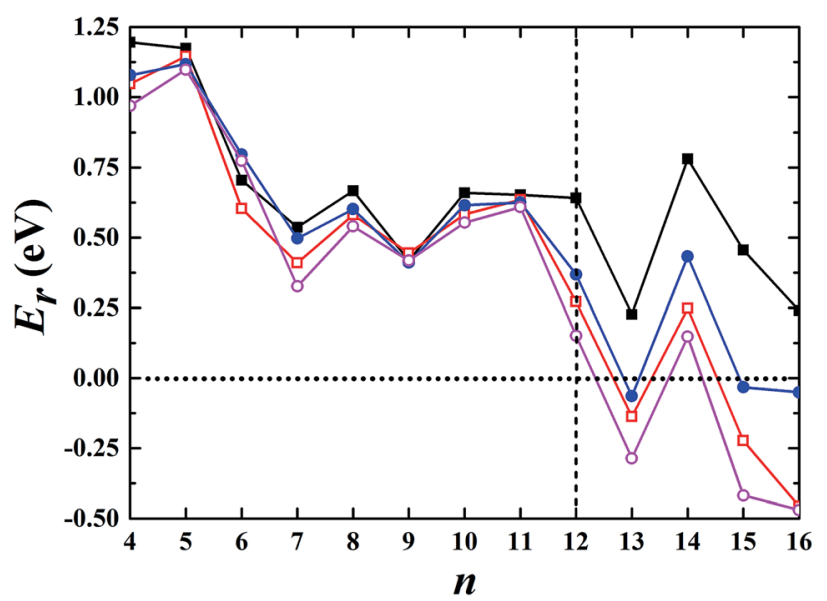

Fig. 1 Relative energy of the selected 3D structure with respect to the corresponding 2D structure. The black solid squares, red empty squares, blue solid circles, and purple empty circles correspond to the data calculated using the PBE, PBEsol, TPSS, and revTPSS functionals, respectively. A positive value indicates a planar geometry as the ground-state structure.

shown in Fig. 1, the PBE results did not show the 2D-3D structural transition with the $2 \mathrm{D}$ structures as the ground state for each cluster, conflicting with the experimental results. For example, the experimental studies indicated a hollow cage structure (see ESI Fig. $\mathrm{S} 5 \dagger$ ) as the ground state for the $\mathrm{Au}_{16}{ }^{-}$ cluster. ${ }^{19}$ However, the PBE functional biased the planar structure as the ground state; its energy was $\sim 0.2 \mathrm{eV}$ lower than that of the first 3D low-energy isomer. TPSS, the advanced semilocal functional, predicted 3D structures as the ground states for the $\mathrm{Au}_{13}{ }^{-}, \mathrm{Au}_{15}{ }^{-}$, and $\mathrm{Au}_{16}{ }^{-}$clusters. However, the prediction of a $2 \mathrm{D}$ ground state structure for the $\mathrm{Au}_{14}{ }^{-}$cluster does not agree with the experimental results. ${ }^{20}$ The PBEsol and revTPSS functionals, which accurately describe the surface energy and are expected to perform better when studying gold clusters, suggested that the 2D-3D structural transition occurred at $n=13$, disagreeing with the experimental results. Although a 3D structure was experimentally determined as the ground state for $\mathrm{Au}_{12}{ }^{-},{ }^{20}$ the total energies of the 2D structures were calculated to be 0.27 and $0.15 \mathrm{eV}$ lower than those of the 3D structures using the PBEsol and revTPSS functionals, respectively.

We also studied the first low-energy 3D structures calculated with the PBE functional for $\mathrm{Au}_{12}{ }^{-}$to facilitate discussion (see ESI Fig. S5 $†$ ). The PBE calculations predict the 2D structure as the ground state with an energy $0.64 \mathrm{eV}$ lower than that of the 3D geometry. Previously, Gong, Wang, and coworkers demonstrated that the temperature may affect the theoretical understanding of the experimental results. ${ }^{21}$ To evaluate the temperature effects by considering the contribution of entropy, we also calculated the free energy of the $\mathrm{Au}_{12}{ }^{-}$cluster via PBE calculations. These calculations were carried out using the calculated total binding energy $\left(E_{0}\right)$ at the PBE level at the zero temperature and the harmonic vibrational entropy as follows: $:^{21,53-55}$

$$
F=E_{0}+k_{\mathrm{B}} T \sum_{q} \sum_{i} \ln \left\{2 \sinh \left(\frac{\hbar \omega_{i}(\boldsymbol{q})}{2 k_{\mathrm{B}} T}\right)\right\},
$$

where $\hbar, k_{\mathrm{B}}, T$, and $\omega_{i}(\boldsymbol{q})$ are Plank's constant, Boltzmann's constant, temperature, and vibration frequency for the $i$ th phonon mode at wave vector $\boldsymbol{q}$ in the Brillouin zone. The calculated free energies (see ESI Fig. S6†) show that the 2D structure remains lower in energy, ruling out the possibility that temperature effects can bridge the difference between the theoretical and experimental results for $\mathrm{Au}_{12}{ }^{-}$. Instead, the discrepancy is likely attributed to the failure to accurately describe the nonlocal energy of the semilocal density functional. 

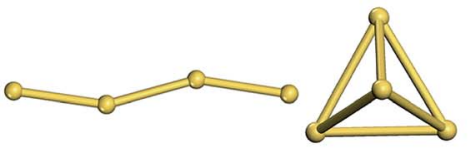

GS

$\mathrm{Au}_{4}^{-}$

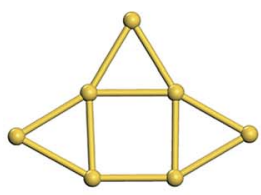

GS

$\mathrm{Au}_{7}^{-}$

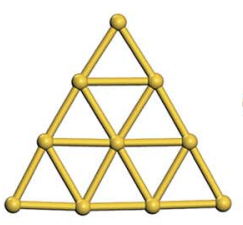

GS

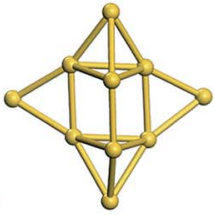

$0.20 \mathrm{eV}$

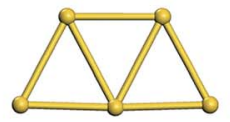

GS

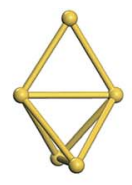

$1.15 \mathrm{eV}$

$\mathrm{Au}_{5}^{-}$

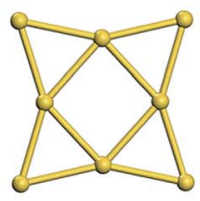

GS

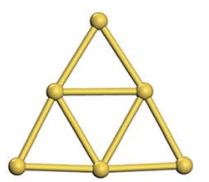

GS

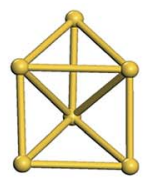

$0.50 \mathrm{eV}$

$\mathrm{Au}_{6}^{-}$

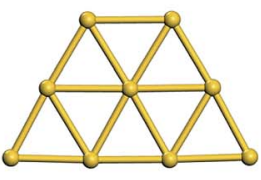

GS

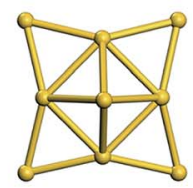

$0.28 \mathrm{eV}$

$\mathrm{Au}_{8}^{-}$
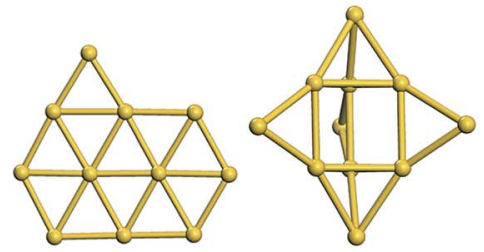

GS

Aus

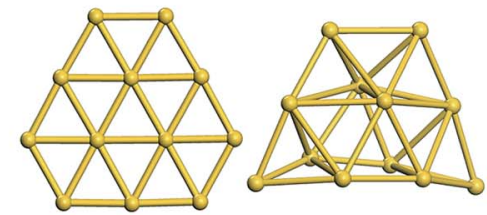

$0.03 \mathrm{eV}$
GS
$\mathrm{Au}_{10}^{-}$

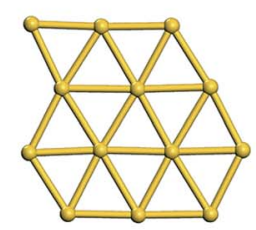

$0.47 \mathrm{eV}$

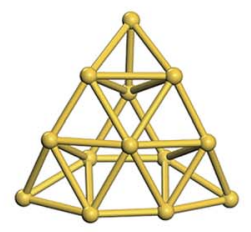

GS

$\mathrm{Au}_{13}^{-}$

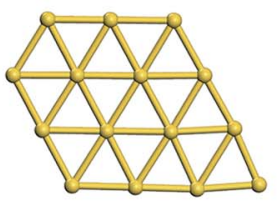

$0.61 \mathrm{eV}$

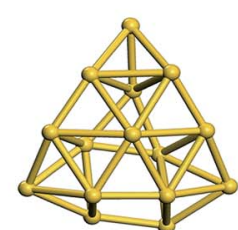

GS

$A \overline{u_{11}}$

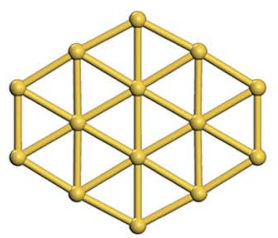

$0.20 \mathrm{eV}$

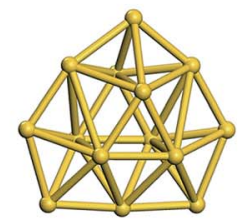

GS

$\mathrm{Au}_{14}^{-}$

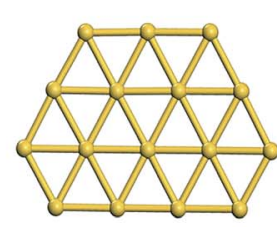

$0.74 \mathrm{eV}$

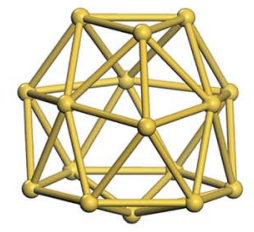

GS

$\mathrm{Au}_{15}^{-}$

$\mathrm{Au}_{16}^{-}$

Fig. 2 The lowest-energy 2D and 3D structures among the corresponding planar and non-planar structures obtained using the TSD-PBE method for $\mathrm{Au}_{n}{ }^{-}$anionic clusters. GS stands for the ground-state structure. The relative energy is provided below the corresponding isomer. A dashed line is added at the experimentally determined 2D-3D structural transition size $n=12$.

\subsection{Nonlocal effects on $\mathrm{Au}_{n}{ }^{-}$clusters}

The vacancy formation enthalpy is a fundamental parameter of metal crystals. Typical semilocal approximations such as PBE fail to yield accurate enthalpies of $\mathrm{Ag}$ and $\mathrm{Au}$, although they give accurate values for $\mathrm{Cu}$. Chen and co-workers ${ }^{37}$ carried out a comprehensive study by mixing $0.1,0.25$, and 0.4 nonlocal exchange interactions to extend the conventional DFT method for the study of the vacancy formation enthalpies of $\mathrm{Cu}, \mathrm{Ag}$, and
Au. The obtained values agree very well with the experimental data, indicting the importance of nonlocality when studying Ag and Au materials. The nonlocal contributions were also found to be critical for studying $\mathrm{Cu}-\mathrm{Au}$ intermetallic alloys, especially those with Au-rich compositions. ${ }^{56}$ Inspired by these findings, we hypothesized that including nonlocal effects in firstprinciples calculations could improve the accuracy of the DFT method for dealing with gold clusters. 
Table 3 VDEs in units of eV calculated using the TSD-PBE method for the structures of gold anions shown in Fig. 2

\begin{tabular}{llllllll}
\hline Anion & $2 \mathrm{D}$ & $3 \mathrm{D}$ & Expt. & Anion & $2 \mathrm{D}$ & $3 \mathrm{D}$ & Expt. \\
\hline $\mathrm{Au}_{4}{ }^{-}$ & 2.73 & 2.65 & 2.75 & $\mathrm{Au}_{11}{ }^{-}$ & 3.66 & 3.10 & 3.80 \\
$\mathrm{Au}_{5}{ }^{-}$ & 3.08 & 2.81 & 3.09 & $\mathrm{Au}_{12}{ }^{-}$ & 3.17 & 3.09 & 3.06 \\
$\mathrm{Au}_{6}{ }^{-}$ & 2.20 & 2.96 & 2.13 & $\mathrm{Au}_{13}{ }^{-}$ & 3.74 & 3.88 & 3.94 \\
$\mathrm{Au}_{7}{ }^{-}$ & 3.44 & 3.60 & 3.46 & $\mathrm{Au}_{14}{ }^{-}$ & 3.77 & 3.03 & 3.00 \\
$\mathrm{Au}_{8}{ }^{-}$ & 2.88 & 2.98 & 2.79 & $\mathrm{Au}_{15}{ }^{-}$ & 3.79 & 3.64 & 3.65 \\
$\mathrm{Au}_{9}{ }^{-}$ & 3.70 & 3.55 & 3.83 & $\mathrm{Au}_{16}{ }^{-}$ & 3.72 & 3.89 & 4.03 \\
$\mathrm{Au}_{10}{ }^{-}$ & 3.87 & 2.87 & 3.91 & - & - & - & - \\
\hline
\end{tabular}

Taking the best $2 \mathrm{D}$ and $3 \mathrm{D}$ structures for $\mathrm{Au}_{12}{ }^{-}, \mathrm{Au}_{16}{ }^{-}$, and $\mathrm{Au}_{20}{ }^{-}$(see ESI Fig. $\mathrm{S}^{\dagger} \dagger$ ) as prototypes, we calculated the corresponding relative energies $E_{\mathrm{r}}$ using the HSE functional. Along with an increase in the hybridization ratio $\alpha$ for the nonlocal exchange interactions, $E_{\mathrm{r}}$ continuously decreased (see ESI Fig. S7 $\dagger$ ). The 3D structure was always lower in energy for the $\mathrm{Au}_{20}{ }^{-}$cluster, while the $2 \mathrm{D}$ one remained the ground-state energy configuration for the $\mathrm{Au}_{12}{ }^{-}$cluster. The 3D structure became the lowest-energy configuration when $\alpha=0.4$ for $\mathrm{Au}_{16}{ }^{-}$. Based on the experimental confirmation of the $3 \mathrm{D}$ ground-state structure of the $\mathrm{Au}_{12}{ }^{-}$cluster, the HSE functional could not properly deal with the gold clusters, although it yielded an accurate vacancy formation enthalpy for bulk gold.

Compared to the HSE functional that incorporates nonlocal exchange energy, the van der Waals density functional (vdWDF), which accounts for the dispersion interaction, can enhance the description of nonlocal effects in a different way. Previous studies on $\mathrm{Na}, \mathrm{Au}$, and $\mathrm{Hg}$ clusters using vdW-DFT carried out by Aguado et $a l^{42}$ and Fernández et al. ${ }^{43}$ suggest that vdW-DFT improves the accuracy of DFT calculations of metal clusters. In these studies, the method proposed by Dion

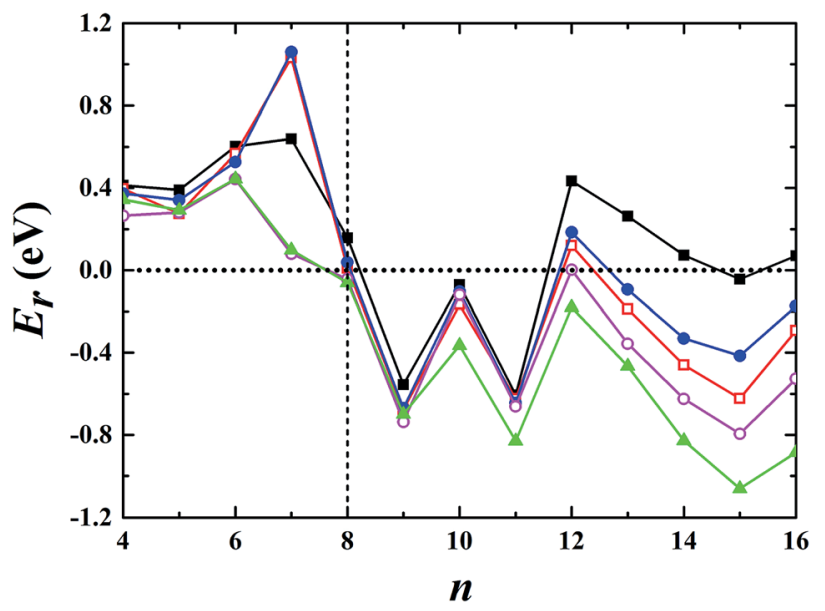

Fig. 4 Relative energy $E_{\mathrm{r}}$ used to determine whether the preferred ground-state structures of $\mathrm{Au}_{n}{ }^{+}$cationic clusters are 3D or 2D geometries. The black solid squares, red empty squares, blue solid circles, purple empty circles, and green solid triangles correspond to the data calculated using the PBE, PBEsol, TPSS, revTPSS, and TSDPBE methods, respectively. A positive value indicates a planar geometry as the ground-state structure.

et $a l .{ }^{57}$ was adopted to account for nonlocal energy by dividing the exchange-correlation energy into three parts:

$$
E_{\mathrm{xc}}[n(r)]=E_{\mathrm{x}}^{\mathrm{GGA}}[n(r)]+E_{\mathrm{c}}^{\mathrm{LDA}}[n(r)]+E_{\mathrm{c}}^{\mathrm{nl}}[n(r)],
$$

where $E_{\mathrm{x}}^{\mathrm{GGA}}[n(r)]$ is the exchange energy of the semilocal GGA exchange functional, $E_{\mathrm{c}}^{\mathrm{LDA}}[n(r)]$ accounts for the correlation energy of the local density approximation (LDA), and $E_{\mathrm{c}}^{\mathrm{nl}}[n(r)]$ is the dispersion interaction energy as the nonlocal correlation energy. Although the vdW-DFT in Dion's functionalism

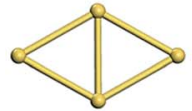

$\mathrm{Au}_{4}^{+}$

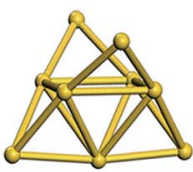

Aus

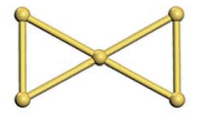

$\mathrm{Au}_{5}^{+}$

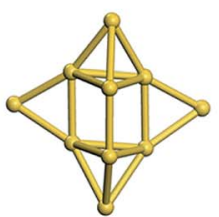

$\mathbf{A u}_{10}^{+}$

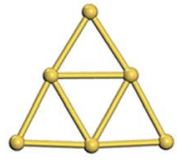

$\mathrm{Au}_{6}^{+}$

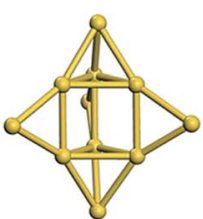

$\mathrm{Au}_{11}^{+}$

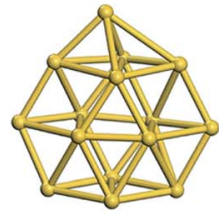

$\mathrm{Au}_{15}^{+}$

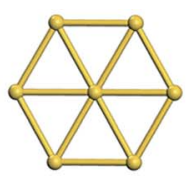

$\mathbf{A u}_{7}^{+}$

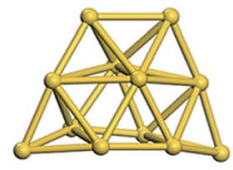

$\mathrm{Au}_{12}^{+}$

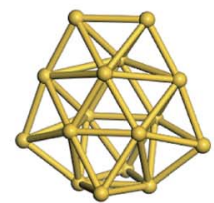

$\mathrm{Au}_{16}^{+}$

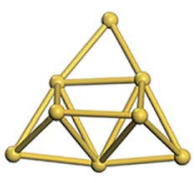

$\mathrm{Au}_{\mathbf{8}}^{+}$

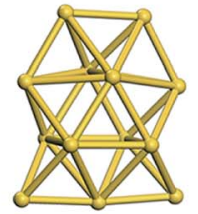

$\mathrm{Au}_{13}^{+}$

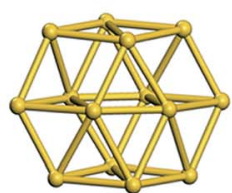

$\mathrm{Au}_{14}^{+}$

Fig. 3 Ground-state structures determined using the TSD-PBE method for $\mathrm{Au}_{n}{ }^{+}$cationic clusters. 

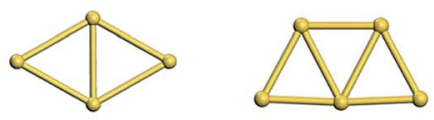

$\mathrm{Au}_{4}$

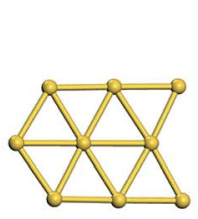

$\mathrm{Au}_{9}$

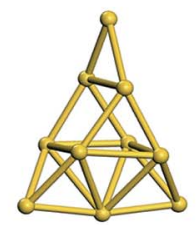

$\mathrm{Au}_{10}$

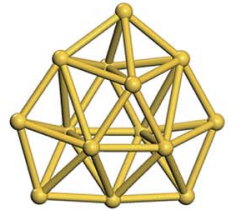

$\mathrm{Au}_{14}$

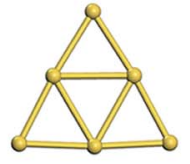

$\mathbf{A u}_{6}$

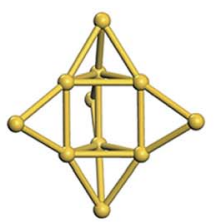

$\mathbf{A u}_{11}$

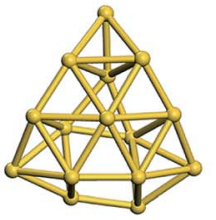

$\mathbf{A u}_{15}$

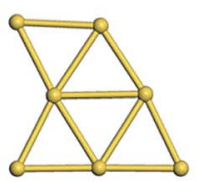

$\mathrm{Au}_{7}$

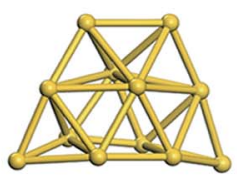

$\mathrm{Au}_{12}$

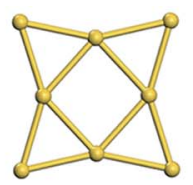

$\mathrm{Au}_{8}$

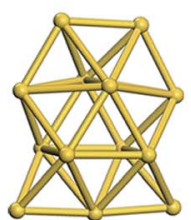

$\mathrm{Au}_{13}$

Fig. 5 The ground-state structures determined using the TSD-PBE method for $\mathrm{Au}_{n}$ neutral clusters.

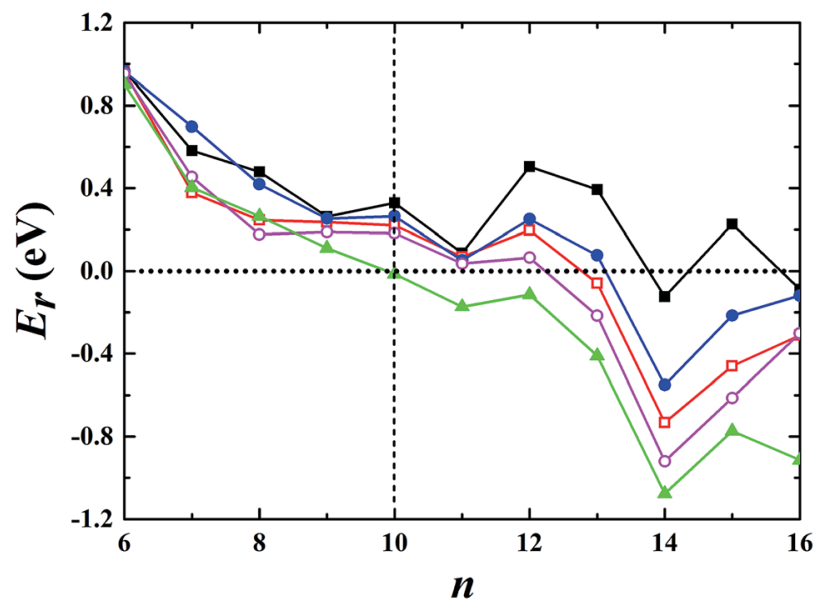

Fig. 6 Relative energy $E_{r}$, as defined by formula (2), indicating whether the preferred ground-state structures of $\mathrm{Au}_{n}$ neutral clusters are 3D or 2D geometries. The black solid squares, red empty squares, blue solid circles, purple empty circles, and green solid triangles correspond to the data calculated using the PBE, PBEsol, TPSS, revTPSS, and TSDPBE methods, respectively. A positive value indicates a planar geometry as the ground-state structure.

improved the DFT results for gold clusters, it still failed to predict the $2 \mathrm{D}-3 \mathrm{D}$ structural transition of $\mathrm{Au}_{12}{ }^{-}{ }^{43}$

Currently, vdW interactions are most commonly accounted for by adding a pairwise interatomic $C_{6} R^{-6}$ term to the DFT energy:

$$
E_{\mathrm{vdW}}=-\frac{1}{2} \sum_{\mathrm{A}, \mathrm{B}} f_{\mathrm{damp}}\left(R_{\mathrm{AB}}, R_{\mathrm{A}}^{0}, R_{\mathrm{B}}^{0}\right) C_{6 \mathrm{AB}} R_{\mathrm{AB}}{ }^{-6},
$$

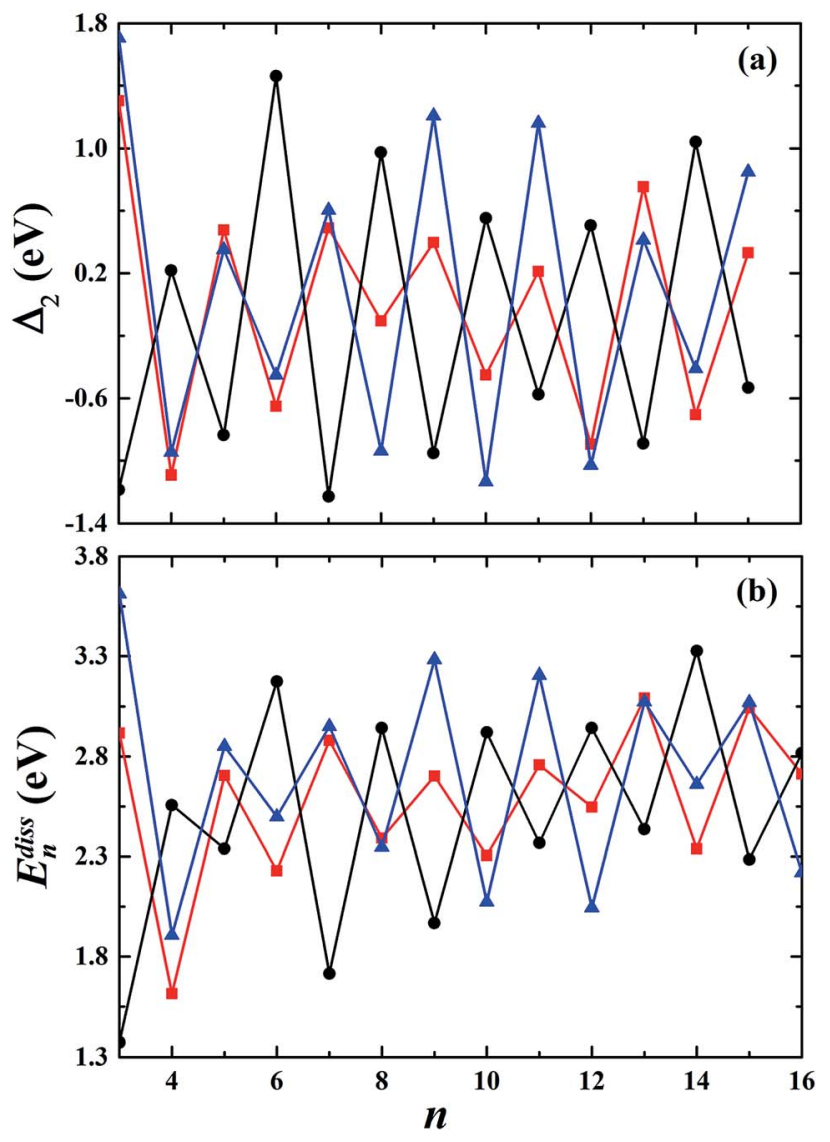

Fig. 7 The calculated second-order finite difference of binding energy $\Delta_{2}$ and the dissociation energy $E_{n}^{\text {diss }}$ of the studied gold clusters. 


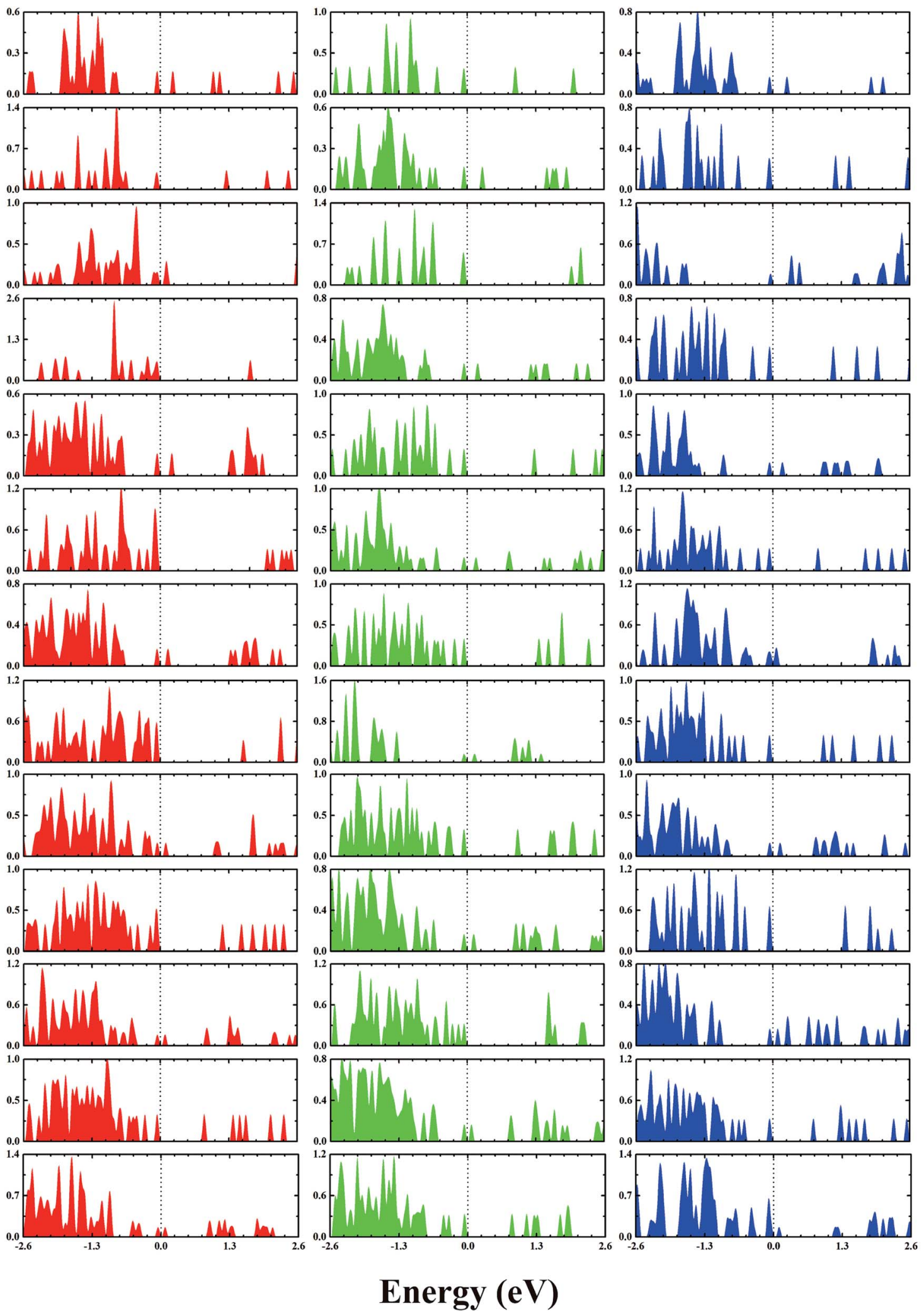

Fig. 8 The calculated densities of states of gold clusters with sizes of $n=4-16$ from the upper row to the lower row, respectively. The red, green, and blue colors correspond to the densities of anion, neutral, and cation clusters, respectively. The Fermi levels are shifted to 0 eV.

where $R_{\mathrm{AB}}$ is the distance between atoms $\mathrm{A}$ and $\mathrm{B}, C_{6 \mathrm{AB}}$ is the corresponding $C_{6}$ coefficient, and $R_{\mathrm{A}}^{0}$ and $R_{\mathrm{B}}^{0}$ are the vdW radii of atoms $\mathrm{A}$ and $\mathrm{B}$, respectively. The $R_{\mathrm{AB}}{ }^{-6}$ singularity at small distances is eliminated by the short-range damping factor $f_{\text {damp }}\left(R_{\mathrm{AB}}, R_{\mathrm{A}}^{0}, R_{\mathrm{B}}^{0}\right)$. Most schemes used to include the vdW interaction in DFT calculation have a serious shortcoming arising 
from their empirical nature. In these approaches, $C_{6} R^{-6}$ is determined by fitting to experimental $C_{6}$ coefficients and/or post-Hartree-Fock binding energy data rather than being obtained directly from the electronic structure. Recently, Tkatchenko and Scheffler ${ }^{\mathbf{4 4}}$ proposed an accurate method to determine the $C_{6}$ coefficients and vdW radii from the mean-field groundstate electronic density of DFT calculations. In their scheme, the Fermi-type damping function was adopted:

$$
f_{\text {damp }}\left(R_{\mathrm{AB}}, R_{\mathrm{AB}}^{0}\right)=\frac{1}{1+\exp \left[-d\left(\frac{R_{\mathrm{AB}}}{s_{\mathrm{R}} R_{\mathrm{AB}}^{0}}-1\right)\right]},
$$

where $R_{\mathrm{AB}}^{0}$ is the sum of the vdW radii of free atoms $\mathrm{A}$ and $\mathrm{B}$, which are obtained from the corresponding electron density contours, and $d$ and $s_{\mathrm{R}}$ are free parameters. The parameter $d$ adjusts the damping function steepness and shows almost negligible effects for $12<d<45$. The choice of $d=20$ was carefully tested and was found to accurately describe the binding energy curves of rare gases and vdW-bonded organic molecule dimers. The parameter $s_{\mathrm{R}}$ remains as the single tunable empirical parameter to control the onset of the vdW correction to the DFT energy. The lager the value of $s_{\mathrm{R}}$, the weaker the contribution of the vdW dispersion interaction to the DFT result. Previously, Tao et al. ${ }^{58}$ and Klimes et al. ${ }^{59}$ showed that vdW forces are indispensable in correctly describing metal solids. Thus, we used bulk metal as a reference to tune the onset contribution of the vdW interactions to PBE calculations. By tuning $s_{R}$, we first calculated the vacancy formation energies of $\mathrm{Cu}, \mathrm{Ag}$, and $\mathrm{Au}$ crystals using the PBE functional with the Tkatchenko-Scheffler dispersion vdW correction. The experimental and HSE results from Chen et al. ${ }^{37}$ along with the values obtained from conventional semilocal exchange-correlation functionals such as PBE, PBEsol, TPSS, and revTPSS are also presented for comparison. The calculated data are tabulated in Table 1. Compared to the experimental and HSE results, neither the conventional GGA functionals (PBE and PBEsol) nor the meta-GGA functionals (TPSS and revTPSS) give consistently accurate results for $\mathrm{Cu}, \mathrm{Ag}$, and $\mathrm{Au}$. The PBE gives accurate values of $a$ and $E_{\mathrm{v}}$ for $\mathrm{Cu}$ but not for $\mathrm{Ag}$ and Au. The surface energy-enhanced PBEsol improves the description for $\mathrm{Ag}$ and $\mathrm{Au}$ but worsens it for $\mathrm{Cu}$. TPSS and revTPSS perform well for the lattice constants of $\mathrm{Cu}, \mathrm{Ag}$, and $\mathrm{Au}$; however, it overestimated the vacancy formation energies $E_{\mathrm{v}}$. As shown in Table 1, including the dispersive interaction in the PBE calculation trough the TSD approach provided accurate vacancy formation energies for $\mathrm{Cu}, \mathrm{Ag}$, and $\mathrm{Au}$ solids. The calculated data show the same trend as Chen's HSE results obtained by turning the hybridization ratio $\alpha$ of the HSE functionals to be $0.10,0.25$, and 0.40 for $\mathrm{Cu}, \mathrm{Ag}$, and $\mathrm{Au}$, respectively. ${ }^{37}$ The effects of nonlocal contributions on the calculation accuracy decreased in the following order: $\mathrm{Au}>\mathrm{Ag}>\mathrm{Cu}$.

Interestingly, the TSD-vdW-corrected PBE functional with the scaling parameter $s_{\mathrm{R}}=0.796$ accurately calculated the vacancy formation energy for Au solid and could be applied to the study of Au clusters, suggesting the crucial role of nonlocal contributions in gold clusters. Unlike our results obtained using PBE, PBEsol, TPSS, revTPSS, and HSE ( $\alpha=0.4)$, TSD-PBE confirmed the 2D-3D structural transition in the $\mathrm{Au}_{12}{ }^{-}$cluster, in agreement with experimental results. Starting from $n=12$, the $\mathrm{Au}_{n}{ }^{-}$clusters prefer the 3D structures. We carefully studied the 2D geometric structures for $\mathrm{Au}_{n}{ }^{-}(4 \leq n \leq 16)$ clusters using the TSD-PBE method, and the lowest-energy structure for each anionic cluster is shown in Fig. 2. Similarly, the best 3D structure for each cluster is also provided in Fig. 2. In addition, the first five low-lying isomers for the clusters with $n \geq 12$ are provided in ESI Fig. S8. $\uparrow$ The vertical detachment energy (VDE) accounting for the minimum energy cost to detach an electron from the cluster anion is often used to assign geometric structure of an anionic cluster through comparison with the theoretical and experimental values. Based on the optimized ground state structures, we evaluated the VDEs of the structures shown in Fig. 2 for $\mathrm{Au}_{n}{ }^{-}(4 \leq n \leq 16)$ anions. VDE was estimated by $E_{\mathrm{vs}}\left(\mathrm{Au}_{n}\right)-E_{\mathrm{GS}}\left(\mathrm{Au}_{n}{ }^{-}\right)$, where $E_{\mathrm{GS}}\left(\mathrm{Au}_{n}{ }^{-}\right)$and $E_{\mathrm{vs}}\left(\mathrm{Au}_{n}\right)$ are the energies of the optimized $\mathrm{Au}_{n}{ }^{-}$anion and the anion geometry at the neutral state, respectively. The VDEs calculated with the TSD-PBE method are presented in Table 3. Within the calculation uncertainty, ${ }^{19,22}$ the VDEs of the 2D structures agree with the experimental data ${ }^{\mathbf{1 9 , 2 0 , 2 5 , 6 0}}$ for smaller clusters, whereas the data for the $3 \mathrm{D}$ structures agree with the experimental values for lager clusters, suggesting that the 2D-3D structural transition occurs at $n=12$. Wang and coworkers experimentally confirmed the coexistence of the $2 \mathrm{D}$ and $3 \mathrm{D}$ structures of the $\mathrm{Au}_{12}{ }^{-}$cluster. ${ }^{20}$ The 3D motif accounts for the larger contribution, suggesting that it has a slightly lower total energy than the $2 \mathrm{D}$ motif. Interestingly, the TSD-PBE calculations predict the $3 \mathrm{D}$ configuration (see Fig. 2) to be $30 \mathrm{meV}$ lower in energy than the 2D structure. Johansson et al. ${ }^{35}$ previously estimated the intensity ratio to be $85: 15$ for $3 \mathrm{D} v s$. 2D structures in anionic gold clusters generated at $100 \mathrm{~K}$, which can be applied to the Boltzmann distribution function to estimate the relative energy of $2 \mathrm{D}$ geometry with respect to the $3 \mathrm{D}$ one. This ratio corresponds to a zero-Kelvin energy difference of $\sim 24 \mathrm{meV}$, in good agreement with the TSD-PBE value of $30 \mathrm{meV}$, indicating the validity of TSD-PBE for treating gold clusters. In addition to the $\mathrm{Au}_{12}{ }^{-}$cluster, another significant gold cluster is the $\mathrm{Au}_{16}{ }^{-}$ anion, which was previously proposed as the first discovered fullerene-like metal cage cluster based on experimental investigations. ${ }^{19}$ In our previous studies carried out at the PBE level, the $2 \mathrm{D}$ structure of the $\mathrm{Au}_{16}{ }^{-}$anion, which had a similar structural configuration to that shown in Fig. 2, was found to be more than $0.2 \mathrm{eV}$ lower in total energy than the cage geometry, ${ }^{22}$ contrary to the experimental observation. The nonlocal contributions-enhanced TSD-PBE method remedies this deficiency; the cage is now found to be $0.74 \mathrm{eV}$ lower in energy than the $2 \mathrm{D}$ structure, ruling out the planar geometry.

\subsection{Cationic gold clusters}

Using global minimum structure searching, we carefully optimized the geometric structures of cationic gold clusters. The ground-state structures obtained with TSD-PBE are schematically presented in Fig. 3. The corresponding optimal 2D and 3D structures calculated with the PBE, PBEsol, TPSS, revTPSS, and TSD-PBE functionals are provided in ESI Fig. S9-S13, $\dagger$ 
respectively. The energies used to illustrate whether the 3D structures are preferable, as defined by formula (2), are presented in Fig. 4. For $\mathrm{Au}_{n}{ }^{+}$, the 2D-3D structural transition is predicted with TSD-PBE to happen at $\mathrm{Au}_{8}{ }^{+}$, in agreement with the experimental results. Through experimental evaluations of ion mobility, Gilb et al. ${ }^{28}$ ruled out the planar geometry of the $\mathrm{Au}_{8}{ }^{+}$cluster. As shown in Fig. 4, the TSD-PBE calculations favor the 3D structure by $61 \mathrm{meV}$. Referring to the Boltzmann distribution, this energy difference accounts for a 3D : 2D intensity ratio of $\sim 1560: 10$ in cluster resource, significantly reducing the possibility of detecting the $2 \mathrm{D}$ structure through ion mobility measurement. The first five low-lying isomers calculated using the TSD-PBE method for $\mathrm{Au}_{n}{ }^{+}(n \geq 8)$ cations are also shown in ESI Fig. S14. $\dagger$ In comparison, the PBE calcula-

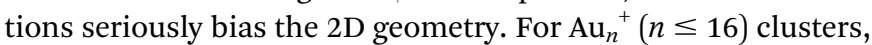
the PBE functional only predicts the $3 \mathrm{D}$ structures as ground states for $\mathrm{Au}_{n}{ }^{+}$with $n=9,10,11$, and 15. For the $\mathrm{Au}_{8}{ }^{+}$cluster, PBE indicates that the 2D structure is $0.16 \mathrm{eV}$ lower in total energy than the 3D geometry. For the PBEsol calculations, the $3 \mathrm{D}$ structure of the $\mathrm{Au}_{8}{ }^{+}$cluster is only $10 \mathrm{meV}$ higher in energy than the 2D structure, which suggests their coexistence, contradicting the experimental results. Beginning at $n=8$, the 3D geometries begin to dominate except at $n=12$, for which the 3D structure is only $0.12 \mathrm{eV}$ higher in energy than the $2 \mathrm{D}$ geometry. Beginning at $n=9$, the TPSS functional starts to favor the 3D configurations for the studied $\mathrm{Au}_{n}{ }^{+}(n \leq 16)$ clusters, with the exception of $n=12$. The revTPSS performs better than the above functionals as it supports the 3D ground structures for $\mathrm{Au}_{n}{ }^{+}(9 \leq$ $n \leq 16$ ) clusters. However, it still biases the $2 \mathrm{D}$ geometry for $\mathrm{Au}_{8}{ }^{+}$, which is $43 \mathrm{meV}$ lower in total energy than the 3D structure. In addition, the calculated energies of the $2 \mathrm{D}$ and $3 \mathrm{D}$ structures of $\mathrm{Au}_{12}{ }^{+}$are almost identical, with the 2D structure being only $2 \mathrm{meV}$ lower in total energy than the 3D structure. The revTPSS results support the 3D geometries as the dominant species in the corresponding clusters, contrary to the experimental findings. In comparison, only the TSD-PBE calculations support the 3D configurations as the ground-state structures for all the studied $\mathrm{Au}_{n}{ }^{+}(8 \leq n \leq 16)$ clusters, again suggesting the importance of nonlocal effects when describing gold clusters.

\subsection{Neutral gold clusters}

For the neutral $\mathrm{Au}_{n}$ clusters, the ground-state structures obtained with the TSD-PBE method are schematically presented in Fig. 5. The 2D-3D structural transition happens at the $\mathrm{Au}_{10}$ cluster. In addition, the low-lying 2D isomers for each cluster were fully optimized. Accordingly, the optimized 2D and 3D geometries obtained with the PBE, PBEsol, TPSS, revTPSS, and TSD-PBE methods are provided in ESI Fig. S15-S19, $\dagger$ respectively. The relative energies, as defined by formula (2), are presented in Fig. 6. The first optimal ground-state 3D structure occurs at $\mathrm{Au}_{14}$ in the PBE and TPSS calculations and at $\mathrm{Au}_{13}$ in the PBEsol and revTPSS calculations. For the $\mathrm{Au}_{15}$ cluster, the PBE calculations still bias the $2 \mathrm{D}$ geometry as the ground-state structure. To the best of our knowledge, there are few experimental studies on the 2D-3D structural transition in neutral gold clusters. However, experimental evidence suggests that the transition occurs at $n=12$ for $\mathrm{Au}_{n}{ }^{-}$clusters and $n=8$ for $\mathrm{Au}_{n}{ }^{+}$ clusters. It seems that more valence electrons would make the $2 \mathrm{D}$ geometry more preferable. Thus, we expect the 2D-3D transition to occur at $8<n<12$. If this is true, calculations using the PBE, PBEsol, TPSS, and revTPSS functionals cannot accurately predict the $2 \mathrm{D}-3 \mathrm{D}$ structural transition for neutral gold clusters. Interestingly, the TSD-PBE method predicts the transition to occur at $n=10$ which seems reasonable (see Fig. 5 and 6). According to the electron counting rule, ${ }^{61}$ small-sized gold clusters can be treated with the Jellium shell closure rule, which describes metal clusters as two subsystems: valence electrons and the positively charged ionic core. The Jellium model of metal clusters applies the spherical potential, which may favor the 3D distribution of metal atoms (e.g., the spherically arranged $\mathrm{Au}$ atoms in the cage structure of $\mathrm{Au}_{16}{ }^{-}$anion). Here, we discuss the typical Jellium model cluster, the $\mathrm{Na}_{n}$ cluster. The atomic radii of $\mathrm{Na}$ and $\mathrm{Au}$ are 1.80 and $1.35 \AA$, respectively. For a given 3D structural configuration, the structure would likely be more compact for $\mathrm{Au}_{n}$ as compared to $\mathrm{Na}_{n}$, which may not be favorable for the $\mathrm{Au}_{n}$ cluster due to its valence electron distribution in a more confined volume. The planar structure is confirmed by both experiment and theory to be preferable for small-sized gold clusters. As the cluster size increases, the number of low-coordination $\mathrm{Au}$ atoms on the corner or edge of the planar configuration also increase, thereby reducing the average coordination number in the planar structure. In contrast, the average coordination number in the $3 \mathrm{D}$ structure likely increase with increasing cluster size, contributing to the increase in total energy compared to the $2 \mathrm{D}$ geometry. This would in turn lead to the 2D-3D structural transition. Compared to the PBE results, the enhanced nonlocal effects help reduce the critical size for the appearance of the $3 \mathrm{D}$ ground-state structure from $n=14$ to $n=10$. Furthermore, the $\mathrm{Au}_{15}$ cluster, for which the $2 \mathrm{D}$ geometry was the lowest-energy configuration in the PBE calculations, is now found to have a 3D ground-state structure in the TSD-PBE calculations. Based on our careful studies, we can conclude that nonlocal effects are important when studying the 2D-3D structural transition for neutral gold clusters, although experimental validation is required. To facilitate the experimental studies, we have provided the first five low-lying isomers for $\mathrm{Au}_{n}(n \geq 10)$ obtained using the TSD-PBE method in ESI Fig. S20. $\dagger$

\subsection{Relative stabilities}

To estimate the relative stabilities of the studied clusters, we calculated the second-order finite difference of binding energy $\Delta_{2}$ and dissociation energy $E_{n}^{\text {diss }}$ as a function of the cluster size $n$, which are respectively defined as:

$$
\Delta_{2}(n)=E_{n+1}-2 E_{n}+E_{n-1},
$$

and

$$
E_{n}^{\text {diss }}=E_{n-1}+E_{1}-E_{n} .
$$

The second-order finite difference of total energy is a general measure of relative stability. Dissociation energy is the energy 
required to evaporate an atom from the cluster, which is also useful to examine the stability. As shown in Fig. 7, the gold clusters exhibit odd-even oscillations in stability, which could be attributed to the electronic stability. Odd-even oscillations were previously observed experimentally. ${ }^{62}$ In addition, a discontinuous variation in cluster ion intensity at peculiar numbers was also observed in experimental mass spectra, in agreement with the magic numbers predicted by a spherically symmetric potential well model. ${ }^{63}$ In Fig. 7, no discontinuous variation is obviously seen in the limited size range of our studied clusters. According to the electron shell structure, when the gold cluster has an even number of valence electrons, it is relatively more stable compared to the cluster with an odd number of valence electrons due to the full filling of molecular orbitals. Fig. 8 presents the calculated density of states (DOS) of the studied anionic, neutral, and cationic gold clusters. The clusters with even numbers of valence electrons have obviously larger band gaps between the highest occupied molecular orbital (HOMO) and the lowest unoccupied molecular orbital (LUMO), supporting their electronic stabilities. In addition, the oscillation agrees with the odd-even reactivity of gold clusters. In experiments, Salisbury et al. ${ }^{29}$ found that the gold cluster with an odd number of electrons could adsorb one molecule of $\mathrm{O}_{2}$ per cluster, whereas the cluster with an even number of electrons showed extremely low or zero reactivity. In the gold cluster with an odd number of electrons, the reactivity was enhanced by the unpaired electron according to the electron shell structure model.

\section{Conclusions}

Our careful studies suggest the importance of nonlocal effects when studying gold materials, in agreement with HSE studies of the vacancy formation energy of bulk gold. In the HSE studies, the nonlocal exchange energy is enhanced, while the PBE correlation energy remains unchanged during the transition; thus, HSE fails to correctly describe the gold clusters. Our calculations with the PBE, PBEsol, TPSS, and revTPSS functionals show that these semilocal functionals also cannot consistently give accurate results for bulk gold and gold clusters. The nonlocal contributions may play a key role. Based on the PBE exchange and correlation energy, the accuracy for calculating both bulk gold and gold clusters could be improved by including the dispersive interaction to enhance the nonlocal contributions. Besides calculating accurately the vacancy formation energy of bulk gold, this method can also remedy the well-known deficiency in which conventional semilocal density functional calculations heavily bias the 2D structures of gold clusters. The 2D-3D structural transitions are found to occur at $\mathrm{Au}_{12}{ }^{-}$for gold anions and $\mathrm{Au}_{8}{ }^{+}$for gold cations, agreeing with the experimental results. Furthermore, the predicted transition at $\mathrm{Au}_{10}$ for neutral clusters seems reasonable, although it requires experimental verification. These findings suggest the importance of nonlocal effects when studying gold and other precious metal materials. This research area remains an important issue for both theoretical and experimental studies. Comprehensive studies on how to accurately account for nonlocal effects as corrections to the widely used semilocal DFT functionals and how much the nonlocal contributions need to be considered in studies of gold and other precious metals are highly needed. By using the vacancy formation energy of bulk gold and the structural transitions of small-sized gold clusters as examples, our study is expected to help stimulate subsequent studies on nonlocal effects.

\section{Conflicts of interest}

There are no conflicts of interest to declare.

\section{Acknowledgements}

The authors acknowledge financial support from the National Natural Science Foundation of China (NSFC) (Grant No. 11674129 and 11374128).

\section{References}

1 M. Haruta, T. Kobayashi, H. Sano and N. Yamada, Chem. Lett., 1987, 16, 405.

2 M. Haruta, Chem. Rec., 2003, 3, 75.

3 T. Ishida and M. Haruta, Angew. Chem., Int. Ed., 2007, 46, 7154 .

4 B. Hammer and J. K. Nørskov, Nature, 1995, 376, 238.

5 M. Haruta, Angew. Chem., Int. Ed., 2014, 53, 52.

6 C. Louis and O. Pluchery, Gold Nanoparticles for Physics, Chemistry and Biology, Imperial College Press, Singapore, 2016.

7 M. Haruta, Nature, 2005, 437, 1098.

8 D. W. Goodman, Nature, 2008, 454, 948.

9 A. A. Herzing, C. J. Kiely, A. F. Carley, P. Landon and G. J. Hutchings, Science, 2008, 321, 1331.

10 M. Turner, V. B. Golovko, O. P. H. Vaughan, P. Abdulkin, A. Berenguer-Murcia, M. S. Tikhov, B. F. G. Johnson and R. M. Lambert, Nature, 2008, 454, 981.

11 B. Yoon, H. Häkkinen, U. Landman, A. S. Wörz, J.-M. Antonietti, S. Abbet, K. Judai and U. Heiz, Science, 2005, 307, 403.

12 P. Gruene, D. M. Rayner, B. Redlich, A. F. G. van der Meer, J. T. Lyon, G. Meijer and A. Fielicke, Science, 2008, 321, 674. 13 P. Pyykkö, Angew. Chem., Int. Ed., 2004, 43, 4412.

14 H. Häkkinen, Chem. Soc. Rev., 2008, 37, 1847.

15 U. Heiz and U. Landman, Nanocatalysis, Springer, New York, 2007.

16 J. Li, X. Li, H.-J. Zhai and L.-S. Wang, Science, 2003, 299, 864. 17 M. Chen and D. W. Goodman, Acc. Chem. Res., 2006, 39, 739. 18 C. Zhang, B. Yoon and U. Landman, J. Am. Chem. Soc., 2007, 129, 2228.

19 S. Bulusu, X. Li, L. S. Wang and X. C. Zeng, Proc. Natl. Acad. Sci. U. S. A., 2006, 103, 8326.

20 W. Huang and L.-S. Wang, Phys. Rev. Lett., 2009, 102, 153401. 21 M. Ji, X. Gu, X. Li, X. G. Gong and L.-S. Wang, Angew. Chem., Int. Ed., 2005, 44, 7119.

22 G. Chen, Q. Wang, Q. Sun, Y. Kawazoe and P. Jena, J. Chem. Phys., 2010, 132, 194306. 
23 Q. Sun, Q. Wang, G. Chen and P. Jena, J. Chem. Phys., 2007, 127, 214706.

24 G. Chen, S. J. Li, Y. Su, V. Wang, H. Mizuseki and Y. Kawazoe, J. Phys. Chem. C, 2011, 115, 20168.

25 F. Furche, R. Ahlrichs, P. Weis, C. Jacob, S. Gilb, T. Bierweiler and M. M. Kappes, J. Chem. Phys., 2002, 117, 6982.

26 X. Xing, B. Yoon, U. Landman and J. H. Parks, Phys. Rev. B: Condens. Matter Mater. Phys., 2006, 74, 165423.

27 M. P. Johansson, I. Warnke, A. Le and F. Furche, J. Phys. Chem. C, 2014, 118, 29370.

28 S. Gilb, P. Weis, F. Furche, R. Ahlrichs and M. M. Kappes, J. Chem. Phys., 2002, 116, 4094.

29 B. E. Salisbury, W. T. Wallace and R. L. Whetten, Chem. Phys., 2000, 262, 131.

30 J. P. Perdew, K. Burke and M. Ernzerhof, Phys. Rev. Lett., 1996, 77, 3865.

31 J. P. Perdew, A. Ruzsinszky, G. I. Csonka, O. A. Vydrov, G. E. Scuseria, L. A. Constantin, X. Zhou and K. Burke, Phys. Rev. Lett., 2008, 100, 136406.

32 J. Tao, J. P. Perdew, V. N. Staroverov and G. E. Scuseria, Phys. Rev. Lett., 2003, 91, 146401.

33 J. P. Perdew, A. Ruzsinszky, G. I. Csonka, L. A. Constantin and J. Sun, Phys. Rev. Lett., 2009, 103, 026403.

34 A. Lechtken, C. Neiss, M. M. Kappes and D. Schooss, Phys. Chem. Chem. Phys., 2009, 11, 4344.

35 M. P. Johansson, A. Lechtken, D. Schooss, M. M. Kappes and F. Furche, Phys. Rev. A, 2008, 77, 053202.

36 A. Glensk, B. Grabowski, T. Hickel and J. Neugebauer, Phys. Rev. X, 2014, 4, 011018.

37 W. Xing, P. Liu, X. Cheng, H. Niu, H. Ma, D. Li, Y. Li and X.-Q. Chen, Phys. Rev. B: Condens. Matter Mater. Phys., 2014, 90, 144105.

38 S.-L. Shang, B.-C. Zhou, W. Y. Wang, A. J. Ross, X. L. Liu, Y.-J. Hu, H.-Z. Fang, Y. Wang and Z.-K. Liu, Acta Mater., 2016, 109, 128.

39 B. Medasani, M. Haranczyk, A. Canning and M. Asta, Comput. Mater. Sci., 2015, 101, 96.

40 J. Heyd, G. E. Scuseria and M. Ernzerhof, J. Chem. Phys., 2003, 118, 8207.

41 Y. Zhang, G. Kresse and C. Wolverton, Phys. Rev. Lett., 2014, 112, 075502.

42 A. Aguado, A. Largo, A. Vega and L. C. Balbás, Chem. Phys., 2012, 399, 252.
43 E. M. Fernández and L. C. Balbás, Phys. Chem. Chem. Phys., 2011, 13, 20863.

44 A. Tkatchenko and M. Scheffler, Phys. Rev. Lett., 2009, 102, 073005.

45 G. Kress and J. Furthmüller, Phys. Rev. B: Condens. Matter Mater. Phys., 1996, 54, 11169.

46 H. J. Monkhorst and J. D. Pack, Phys. Rev. B: Solid State, 1976, 13, 5188.

47 J. Ho, K. M. Ervin and W. C. Lineberger, J. Chem. Phys., 1990, 93, 6987.

48 E. M. Fernández, J. M. Soler, I. L. Garzón and L. C. Balbás, Phys. Rev. B: Condens. Matter Mater. Phys., 2004, 70, 165403.

49 A. R. Oganov and C. W. Glass, J. Chem. Phys., 2006, 124, 244704.

50 A. R. Oganov, A. O. Lyakhov and M. Valle, Acc. Chem. Res., 2011, 44, 227.

51 A. O. Lyakhov, A. R. Oganov, H. T. Stokes and Q. Zhu, Comput. Phys. Commun., 2013, 184, 1172.

52 X.-F. Zhou, X. Dong, A. R. Oganov, Q. Zhu, Y. Tian and H.-T. Wang, Phys. Rev. Lett., 2014, 112, 085502.

53 K. Huang, Statistical Mechanics, Wiley, New York, 2nd edn, 1987.

54 R. E. Allen and F. W. de Wetter, Phys. Rev., 1969, 179, 873.

55 J. Xie, S. P. Chen and J. S. Tse, Phys. Rev. B: Condens. Matter Mater. Phys., 1999, 60, 9444.

56 Y. Zhang, G. Kresse and C. Wolverton, Phys. Rev. Lett., 2014, 112, 075502.

57 M. Dion, H. Rydberg, E. Schröder, D. C. Langreth and B. I. Lundqvist, Phys. Rev. Lett., 2004, 92, 246401.

58 J. Tao, P. Perdew and A. Ruzsinszky, Phys. Rev. B: Condens. Matter Mater. Phys., 2010, 81, 233102.

59 J. Klimes, D. R. Bowler and A. Michaelides, Phys. Rev. B: Condens. Matter Mater. Phys., 2011, 83, 195131.

60 H. Häkkinen, B. Yoon, U. Landman, X. Li, H.-J. Zhai and L.-S. Wang, J. Phys. Chem. A, 2003, 107, 6168.

61 P. Jena and Q. Sun, Chem. Rev., 2018, 118, 5755-5870.

62 I. Katakuse, T. Ichihara, Y. Fujita, T. Matsuo, T. Sakurai and H. Matsuda, Int. J. Mass Spectrom. Ion Processes, 1985, 67, 229.

63 W. D. Knight, K. Clemenger, W. A. de Heer, W. A. Saunders, M. Y. Chou and M. L. Cohen, Phys. Rev. Lett., 1984, 52, 2141. 